\title{
Computationally-Scalable Motion Estimation Algorithm for H.264/AVC Video Coding
}

\author{
Man-Yau Chiu and Wan-Chi Siu, Senior Member, IEEE
}

\begin{abstract}
This paper presents a computationally-scalable motion estimation (ME) algorithm in which it can dynamically control the number of operations spent on ME while the compressed video quality, in terms of PSNR and bit rate, will be scaled up or down smoothly. Unlike fast search algorithms, our proposed algorithm employs a closed-loop control to adaptively adjust the search strategy so that the actual ME computational complexity can be kept close to the allocated budget as much as possible. Experimental results show that when the ME computational budget falls to half of the average number of operations required by the fast full search (for the best quality), on average, the PSNR is reduced by $0.046 \mathrm{~dB}$ and the bit rate is increased by $2.34 \%$ as compared to that of full search for 1920x1080 video sequences and this computationally-scalable ME algorithm is particularly suitable for consumer devices to realize real-time computational budget control for video compression.
\end{abstract}

Index Terms - H.264/AVC, video coding, motion estimation, computationally-scalable.

\section{INTRODUCTION}

H.264/AVC [1], [2] is the latest video coding standard which can achieve higher compression efficiency than that of MPEG-2 at the expense of higher ME computational requirement. Hence, various fast algorithms have been proposed in literature to speed up H.264/AVC ME [6]-[8].

In [6], a fast full search algorithm is proposed in which it adaptively adopts successive elimination algorithm (SEA) [3] and partial distortion search (PDS) [4] for H.264/AVC ME. In [7], a fast block mode determination algorithm is proposed to determine the optimal block mode to code a macro-block (MB). In [8], another fast algorithm is proposed in which it adaptively adjusts the search window size for checking reference frames other than the temporal nearest one.

Ideally, full search or fast full search should be used for ME so that the best compressed video quality can be achieved. But, for practical realization of video encoder for consumer products, e.g. mobile multimedia phone, it is desirable that the video encoder can compress video frames within certain preallocated computational budget as the computational power of these consumer products would be limited.

\footnotetext{
${ }^{1}$ This work is supported in part by the PolyU (CSP) under the Research Grant Council of the Hong Kong SAR Government, PolyU5267/07E.

M.Y. Chiu and W.C. Siu are with the Department of Electronic and Information Engineering, The Hong Kong Polytechnic University, Hung Hon, Kowloon, Hong Kong (e-mail: 03743292g@polyu.edu.hk, and enwcsiu@polyu.edu.hk ).
}

Original manuscript received February 17, 2010

Revised manuscript received April 2, 2010

Current version published 0629 2010;

Electronic version published 07062010 .
In particular, when H.264/AVC video coding is used, the $\mathrm{ME}$ process would be computational intensive and such consumer products may not have sufficient computational power allocated for best-quality video coding all the time.

In order to meet the computational budget requirement for video coding, the simplest way to achieve it is to use fast search for ME. In doing so, though we may meet the computational budget requirement, the compressed video quality may degrade significantly. Such significant degradation in compressed video quality would be due to inefficient utilization of the available computational budget for ME. For example, if the fast search strategy employed is too "aggressive" in reducing the computational complexity, then the allocated computational budget may not be fully utilized and this may result in sub-optimal ME results. The better way to meet the computational budget requirement is to employ computationally-scalable ME algorithm so that the number of operations spent on $\mathrm{ME}$ can be scaled to meet budget requirement.

The common approach to achieve computational scalability in $\mathrm{ME}$ is to adjust certain search parameters, e.g. search range, so as to control the number of operations spent on ME [9][12]. For these ME algorithms, when more computational budget is available, a more accurate $\mathrm{ME}$ will be performed through the use of a more accurate distortion measure, a larger search space and etc. In [9], Lengwehasatit and Ortega proposed to check partial sum of absolute difference (partial SAD, PSAD) at each candidate location and then immediately reject those candidates with PSAD greater than certain threshold value, in which this threshold value can be scaled according to the computational budget available. In [10], Yang et al. proposed to vary the number of predicted motion vectors (MV) and the number of recursive search steps in small diamond search to achieve different computational complexity in ME whereas in [11], Liu, Zhuang, and Dai proposed to vary the maximum searching distance and the maximum searching steps for each search pattern to achieve computational scalability. In [12], Zhang et al. proposed to use different block mode pre-selection methods for reference frames other than the temporal nearest one to achieve computationally scalability in H.264/AVC ME.

Besides this common approach, there are some other approaches proposed in literature to achieve computational scalability in ME. For example, in [13], Mietens, With, and Hentschel presented a ME algorithm in which the computational-scalability is achieved by scaling the motion vector fields approximation using multiple temporal 
references and scaling the number of predicted MV to be evaluated. In [14], Yang, Cai, and Li suggested to use the search priority to determine how the computational resources can be dynamically allocated to each MB, whereas in [15], Tan et al. proposed to use Rate-Distortion (R-D) cost as a measure for dynamic computational resources allocation.

In this paper, a novel computationally-scalable $\mathrm{ME}$ algorithm is proposed. The main objective of this algorithm is to allow video encoder to control the number of operations spent on $\mathrm{ME}$ so that the video coding process can be completed within the pre-allocated computational budget and at the same time, the compressed video quality will be scaled up or down smoothly when the ME computational-complexity has been changed. In particular, when less computational budget is allocated, the compressed video quality will be degraded gracefully. Unlike conventional fast algorithms, our algorithm incorporates a closed-loop control to determine the appropriate search strategy to be used under different computational constraints. Depending on the allocated computational budget and the current $\mathrm{ME}$ computational complexity, the proposed algorithm can dynamically adjust its search strategy, either adopting a fast full search approach or a combination of different fast search techniques, to produce the optimal predicted frame. For example, when the current ME computational complexity is consistently lower than the allocated computational budget, a less "lossy" search strategy would be used so that the computational resources would be better utilized for achieving better ME results. Experimental results show that for coding 1920x1080 video sequences, if we impose a ME computational constraint (for coding a frame) to be half of the average number of computations required by the un-constrained fast full search [6], the proposed algorithm can achieve this new computational requirement within 40 frame-time while the PSNR and bit rate performances are just slightly degraded.

This paper is organized as follows. At first, a brief review of H.264/AVC ME and its fast search algorithms are described in section II. Next, our proposed computationally-scalable ME algorithm is described in section III. Experimental results showing the compression performance of the proposed algorithm under different computational constraints are described in section IV. Finally, a conclusion is given in section $\mathrm{V}$.

\section{REVIEW OF H.264/AVC MOTION ESTIMATION}

Block-based motion estimation/compensation has been widely adopted in various video coding standards, such as MPEG-1, MPEG-2, MPEG-4, H.261, H.263 and etc. Blockbased $\mathrm{ME}$ is a process to find a $\mathrm{MB}$ in the reference frame which is "best" matched (in terms of matching difference criterion) to a target $\mathrm{MB}$ in current frame. Through motion compensation, the target $\mathrm{MB}$ under consideration can be predicted by such "best" matched MB available in the reference frame and the encoder only needs to code the difference between these $\mathrm{MBs}$, i.e. the motion compensated residual. As a result, the temporal redundancy between successive video frames can be reduced.
For block-based ME, the sum of absolute difference (SAD) is commonly used as a criterion to measure the matching difference. Let us consider a target block in the current frame at position $(0,0)$. Then, $\mathrm{SAD}$ between this target $N \mathrm{x} N$ block and a candidate $N \mathrm{x} N$ block at position $(u, v)$ in the reference frame (with reference frame index $z$ ) is defined as follows:

$$
S A D(u, v ; z)=\sum_{j=0}^{N-1} \sum_{i=0}^{N-1}\left|f_{c}(i, j)-f_{z}(u+i, v+j)\right|
$$

where $f_{c}(\cdot, \cdot)$ and $f_{z}(\cdot, \cdot)$ represent pixel intensities in the current frame and reference frame $z$ respectively.

Full search algorithm (FSA) calculates the comparison criterion (e.g. SAD) at each possible location in the search window and then determines which candidate block within the search window can achieve minimum SAD with the target block. In this way, the best matching block within the search window can be obtained.

In H.264/AVC video coding standard, it has added flexibility in the selection of block sizes for motion estimation and compensation. So, for every target $\mathrm{MB}$ under consideration, it can be predicted by one of the following four ways: either by one $16 \times 16 \mathrm{MB}$ partition, two 16x8 MB partitions, two $8 \times 16 \mathrm{MB}$ partitions or four $8 \times 8$ sub-MB partitions from the reference frame as shown in Fig. 1. In particular, if four $8 \times 8$ sub-MB partitions mode is selected, each $8 \times 8$ sub-MB partition can also be further predicted by one of the following four ways: either by one $8 \times 8$ sub-MB partition, two $8 \times 4$ sub-MB partitions, two $4 \times 8$ sub-MB partitions or four $4 \times 4$ sub-MB partitions as shown in Fig. 2. So, totally, there are $3+4^{4}=259$ possible block mode combinations for predicting a target $\mathrm{MB}$.

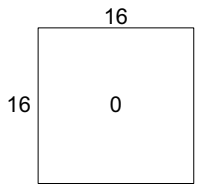

$16 \times 16$ Block Mode 1

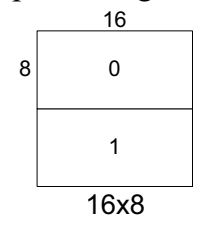

Block Mode 2

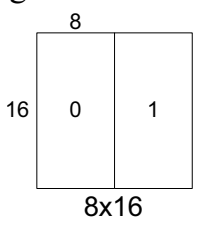

Block Mode 3

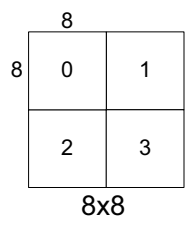

Block Mode 4
Fig. 1. MB partitions: 16x16, 16x8, 8x16, 8x8.

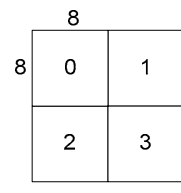

$8 \times 8$

\begin{tabular}{|l|l|}
\multicolumn{3}{|l|}{8} \\
\hline 0 & 1 \\
\hline 2 & 3 \\
\hline 4 & 5 \\
\hline 6 & 7 \\
\hline
\end{tabular}

$8 \times 4$

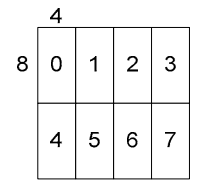

$4 \times 8$
Block Mode 6

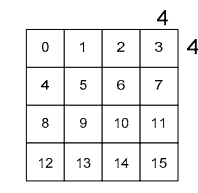

$4 \times 4$

Block Mode 7
Fig. 2. Sub-MB partitions: $8 \times 8,8 \times 4,4 \times 8,4 \times 4$.

Furthermore, in H.264/AVC, for coding a P-macroblock, it is allowed to use more than one previously coded frame as reference for motion-compensated prediction, i.e. for a target $\mathrm{MB}$ in current frame, its MB partitions or sub-MB partitions can be predicted by MB partitions or sub-MB partitions from different reference frames (with the limitation that for those $8 \times 4 / 4 \times 8 / 4 x 4$ sub-MB partitions within the same $8 \times 8$ sub-MB partition, all these sub-MB partitions should be predicted from the same reference frame). 
With such flexibility in choosing the block size and reference frames for motion estimation and compensation, there is a higher chance to find a better prediction to the target MB than the case when the block size is fixed and only one reference frame can be used. But, in doing so, the ME computational complexity will be increased significantly.

In order to speed up this variable block size (VBS) multiple reference frames (MRF) motion estimation, various fast algorithms have been proposed. These fast algorithms can be classified into two groups. One is the lossless in which these algorithms can achieve exactly the same compressed video quality and at the same time with less computation as compared to that of FSA. The other one is the lossy in which these algorithms usually can achieve an even faster speed than that of the lossless algorithms at the expense of some degradation in compressed video quality. Some examples of these fast algorithms are described as follows.

\section{A. Fast Lossless Approach}

1) Application of SEA for H.264/AVC Motion Estimation Successive elimination algorithm (SEA) is a well-known fast full search technique for conventional block-based ME. Here, at each candidate location, a computationally less expensive measure, boundary value $B V=|C-R(u, v ; z)|$, is computed first where $C$ and $R(u, v ; z)$ are the sum norms of the target block and the candidate block respectively and they are defined as follows:

$$
C=\sum_{j=0}^{N-1} \sum_{i=0}^{N-1} f_{c}(i, j), \quad R(u, v ; z)=\sum_{j=0}^{N-1} \sum_{i=0}^{N-1} f_{z}(u+i, v+j)
$$

According to [3], if $B V \geq \mathrm{SAD}_{\min }$, where $\mathrm{SAD}_{\text {min }}$ is the minimum SAD found so far, then it is impossible for this candidate block to achieve the SAD value smaller than $\mathrm{SAD}_{\min }$ and so this candidate should be rejected immediately. In doing so, full SAD computation can be avoided in rejecting impossible candidates. Only when the candidate with $B V$ smaller than $\mathrm{SAD}_{\min }$, then the actual $\mathrm{SAD}$ or partial $\mathrm{SAD}$ (PSAD) will be computed to determine whether it is a better match or not.

In H.264/AVC, the target MB can be partitioned into smaller blocks for motion compensation. So, for full search, we need to perform ME for blocks of different sizes. In [6], Chiu and Siu proposed a way to adaptively adopts SEA for this VBS motion estimation and it is done by modifying the $B V$ computation (or in particular for $C$ and $R(u, v ; z)$ ) according to different block sizes under consideration. By further exploiting the characteristics of VBS ME and slight modification in the definition of SEA boundary value, boundary value reuse is also proposed in [6] in which the boundary values computed for smaller block mode can be reused to obtain the boundary values of larger block mode. In doing so, it can significantly reduce the amount of operations required in computing the SEA boundary values of different block sizes.

\section{B. Fast Lossy Approach}

1) Fast H.264/AVC block mode decision by using feature extracted from current frame

For H.264/AVC VBS motion estimation, the ME process can be speeded up if we can quickly determine which block mode is optimal for motion compensation without actual evaluation of all the block modes. In [7], a fast algorithm is proposed in which it utilizes the spatial homogeneous and temporal stationary properties of the current block for fast block mode decision. Here, the edge map of the current frame is used as the feature to determine whether the $\mathrm{MB}$ is homogenous or not while the MB difference between the current MB and the collocated MB in the previous frame is used as the feature to determine whether the $M B$ is in stationary region or not. If the current $\mathrm{MB}$ (or $8 \times 8 \mathrm{MB}$ partition) is found to be in homogenous or stationary region, block mode 1 (or 4) will be used. In doing so, if the block mode can be quickly determined, ME process can be speeded up since much of the smaller block mode evaluations can be skipped.

\section{2) Reduction of search space in temporal domain by} using adaptive search window size

In [8], a fast search technique is proposed for multiple reference frame ME. Here, the full search window is used for finding the best match in the temporal nearest reference frame. Let $L$ be the length of the motion vector found in this temporal nearest frame. As it was found in [8] that the probability to obtain an even better match in other reference frames is low and the probability that the length of such better MV is greater than $L$ is low too, so the ME process can be speeded up by reducing the size of search window to $L$ for the rest of the reference frames. In doing so, the compressed video quality will not be greatly affected as the chance to miss the better match in other reference frames is low.

\section{Proposed Algorithm}

In order to meet the allocated computational budget requirement for video compression, the proposed algorithm can employ the appropriate search strategy for ME. For example, if less computational budget is allocated, this algorithm will tend to adjust its search strategy to be more "lossy" so that less computation will be required. In doing so, a less optimal match would be used for motion compensation. On the other hand, if more computational budget is allocated, this algorithm will tend to perform more like a "full" search so that a better match would be found. Totally, there are five search strategies available and if a particular search strategy is used, the algorithm is said to be operating at one particular ME operating state, ranging from state 1 to 5 , The higher is the ME operating state, a more "lossy" search approach is used and hence less computation will be required. Here, a closed-loop control is used to monitor the ME process in coding each frame and then determine the appropriate search strategy or the ME state to be used. The proposed ME 
operating states and the control mechanism are described in the following two sub-sections.

\section{A. Motion Estimation Operating States}

The proposed ME algorithm can operate at one of the following ME states, in which at each operating state, a different fast search strategy is used.

1) $M E$ state 1: In this state, a fast full search algorithm as described in [6] is used. Let us denote this fast full search algorithm as H.264 lossless SEA+PDS algorithm.

2) $M E$ state 2: In this state, a lossy version of [6] is used. Here, the SEA boundary value $(B V)$ computation is simplified in which the block sum norms $(C$ and $R(u, v ; z))$ is obtained by summing alternate pixels within the block (i.e. 2:1 decimation is employed in computing the sum norms) and such precomputed $B V$ are used "as is" the SAD in finding the optimal candidate. In other words, the candidate block that can achieve smallest $B V$ (instead of SAD) with the target block will be directly selected as the optimal match. Let us denote this fast lossy algorithm as H.264 lossy SEA+PDS algorithm.

3) $M E$ state 3: In this state, H.264 lossy SEA+PDS is used together with the early termination in finding the optimal reference frame(s) to code a target MB. In H.264/AVC reference software [16], there are two loops used in $\mathrm{ME}$ process to find the best match. The outer loop is for traversing block modes and the inner loop is for traversing reference frames. Such ME looping structure (for block mode 1, 2, and 3 ) is shown in Fig. 3.

When a particular $\mathrm{MB}$ partition is considered, all the candidates from all reference frames will be evaluated. At this operating state, we can terminate the inner loop earlier if the $\mathrm{SAD}$ of the optimal candidate found in a particular reference frame is smaller than certain threshold value. If such candidate can be found, then the search process for this MB partition will be terminated at this reference frame and the rest of the reference frames will not be checked.

Let $T^{R}{ }_{i}$ be the threshold for block mode $i$ reference frame early termination, where $i=1,2, . ., 7$. For example, when finding the best match for target 16x8 MB partition (mode 2), if the optimal candidate $16 \times 8 \mathrm{MB}$ partition in reference frame 2 is found to have the SAD value less than $T^{R}{ }_{2}$, the search process will not continue to check the $16 \times 8$ candidates in reference frames with reference frame index greater than 2 . Instead, the next block partition or block mode will be checked.

If a larger threshold is used, there will be a higher chance to terminate the inner loop earlier. To provide a gradual change in early termination criterion, 13 sub-states are defined with increasing threshold values used for the reference frame early termination. At sub-state 13, the threshold values for each block mode are $T^{R}{ }_{1,13}=1024, T_{2,13}^{R}=512, T_{3,13}^{R}=512, T_{4,13}^{R}$ $=256, T^{R}{ }_{5,13}=128, T_{6,13}^{R}=128$ and $T^{R}{ }_{7,13}=64$. At sub-state $S$, where $S=1,2, . ., 12$, the threshold values, $T_{i, S}^{R}$, are defined as follows:

$$
T^{R}{ }_{i, S}=\frac{S}{13} T^{R}{ }_{i, 13}
$$

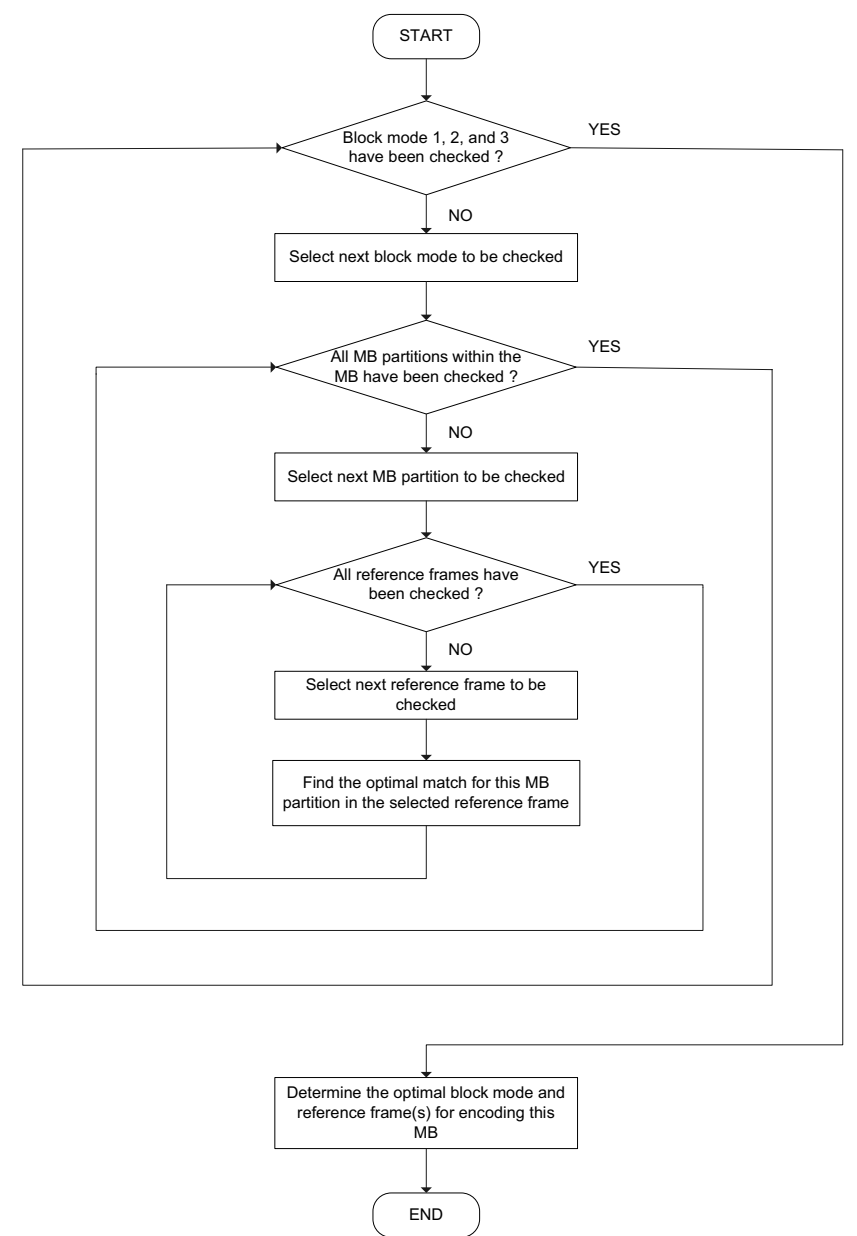

Fig. 3. H.264/AVC reference software ME looping structure (for block mode 1,2 , and 3 ).

4) ME state 4: In this state, the H.264 lossy SEA+PDS is used together with the early termination in finding the optimal reference frame as well as the optimal block mode to code a target $\mathrm{MB}$, i.e. early termination is introduced at both outer and inner loops. For reference frame early termination (for inner loop), maximum threshold values, $T^{R}{ }_{i, 13}$, are used. For block mode early termination (outer loop), once the SAD value achieved in coding a target $\mathrm{MB}$ or $\mathrm{MB}$ partitions by a particular block mode is found to be smaller than a certain pre-defined threshold, the search process will not check the rest of the block modes and this optimal block mode will be used to code the target block.

Again, let $T^{B}{ }_{i}$ be the threshold for block mode $i$ early termination, where $i=1,2, . ., 7$. For example, if the MB is coded by block mode 2 and the optimal SAD found for both 16x8 MB partitions are smaller than $T^{B}{ }_{2}$, then this MB will be coded by block mode 2 and the search process will not check the remaining block modes $(3-7)$.

Similar to the reference frame early termination in ME state 3, 13 sub-states are defined with increasing threshold values used for block mode early termination. At sub-state 13, the threshold values for each block mode are $T^{B}{ }_{1,13}=1024, T_{2,13}^{B}$ $=512, T_{3,13}^{B}=512, T_{4,13}^{B}=256, T_{5,13}^{B}=128, T_{6,13}^{B}=128$ and $T^{B}{ }_{7,13}=64$. At sub-state $S$, where $S=1,2, . ., 12$, the threshold 
values, $T^{B}{ }_{i, S}$, are defined as follows:

$$
T^{B}{ }_{i, S}=\frac{S}{13} T^{B}{ }_{i, 13}
$$

5) ME state 5: In this state, similar search techniques as in $\mathrm{ME}$ state 4 are used. Here, maximum threshold values, $T^{R}{ }_{i, 13}$ and $T^{B}{ }_{i, 13}$, are used for reference frame (inner loop) and block mode (outer loop) early termination respectively. Besides these, an adaptively adjusted search window size [8] is also used for checking the reference frames other than the temporal nearest one.

If a smaller search window is used for farther reference frames, the ME process can be completed earlier. Hence 13 sub-states are defined with decreasing search window size used for checking farther reference frames. Let $S R$ be the original search range; and $L$ be the length of the motion vector found in the temporal nearest reference frame and this also represents the smallest search range that can be used for other reference frames. Then the reduced search range, $S R_{\text {reduced, } S}$, to be used at sub-state $S$, where $S=1,2,3, . ., 13$, are defined as follows:

$$
S R_{\text {reduced }, S}=S R-\frac{(S R-L)}{13} S
$$

\section{B. Core Procedure of the Proposed Algorithm}

Let $C_{T}$ be the target ME computational budget allocated to code each video frame and $C_{i}$ be the actual number of operations required by the ME algorithm to code $i^{\text {th }}$ video frame. The objective of control mechanism is trying to adjust the search strategy so that the average ME operations within a moving window to be as close to $C_{T}$ as possible. Here, at current frame $j$, the moving window is defined as the last $W$ frames running from $(j-W+1)$ to $j$ and the average computational complexity within this window, $C_{W}$, is defined as follows:

$$
C_{W}=\frac{1}{W} \sum_{i=j-W+1}^{j} C_{i}
$$

So, if it is found $C_{W}$ exceeds certain tolerance limit, the control mechanism will drive the ME process to a higher operating state (or sub-state), i.e. a faster search strategy should be used in coding subsequent frames and vice versa.

Here, let $\gamma_{i}$ be the budget utilization in coding frame $i$ and $\gamma_{i}$ is defined as follows:

$$
\gamma_{i}=\frac{C_{i}}{C_{T}}
$$

and it can be used as a measure to indicate how efficient the computational budget is utilized.

The pseudo-code for determining the transition between different ME states is described as follows:

Step 1: After coding the $j^{\text {th }}$ video frame, the following $x_{1}, x_{2}$ and $C_{\text {avg }}$ are computed:

$$
\begin{aligned}
& x_{1}=\frac{1}{3} \sum_{i=j-5}^{j-3} C_{i}, \quad x_{2}=\frac{1}{3} \sum_{i=j-2}^{j} C_{i} \\
& C_{\text {avg }}=\frac{1}{2}\left(x_{1}+x_{2}\right)
\end{aligned}
$$

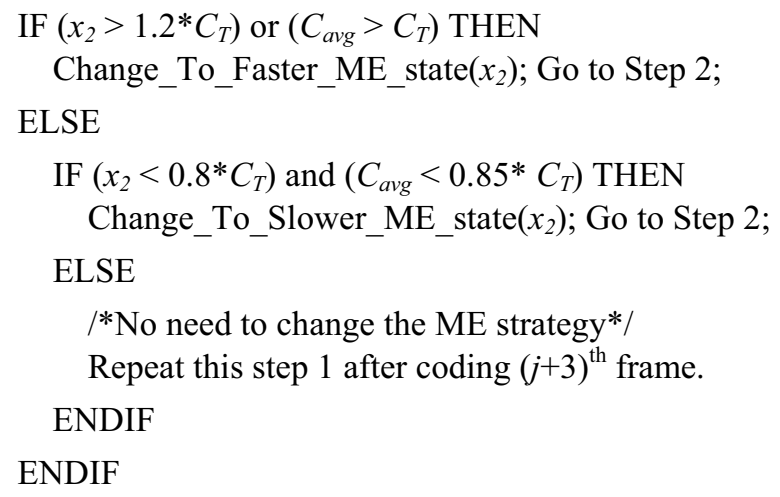

Step 2: After changed the ME state or sub-state, six more frames will be coded. After coding $(j+6)^{\text {th }}$ frame, the following $x_{3}, x_{4}$ and $C_{a v g}$ ' are computed:

$$
\begin{aligned}
& x_{3}=\frac{1}{3} \sum_{i=j+1}^{j+3} C_{i}, \quad x_{4}=\frac{1}{3} \sum_{i=j+4}^{j+6} C_{i} \\
& C_{\text {avg }}{ }^{\prime}=\frac{1}{2}\left(x_{3}+x_{4}\right)
\end{aligned}
$$

IF (ME state has been changed to faster state previously) THEN

$$
\begin{aligned}
& \text { IF }\left(\left(x_{2}>x_{3}\right) \text { and }\left(x_{2}>x_{4}\right)\right) \text { or }\left(C_{\text {avg }}>C_{\text {avg }}\right) \text { THEN } \\
& \text { Go to step 1; } \\
& \text { ELSE } \\
& \text { Change_To_Faster_ME_state }\left(x_{4}\right) \text {; } \\
& \text { Repeat step } 2 \text { after coding }(j+12)^{\text {th }} \text { frame; } \\
& \text { ENDIF } \\
& \text { LSE } \\
& \text { /* ME state has been changed to slower state } \\
& \text { previously } * \text { / } \\
& \text { IF }\left(\left(x_{2}<x_{3}\right) \text { and }\left(x_{2}<x_{4}\right)\right) \text { or }\left(C_{\text {avg }}<C_{\text {avg }}\right) \text { THEN } \\
& \text { Go to step 1; } \\
& \text { ELSE } \\
& \text { Change_To_Slower_ME_state }\left(x_{4}\right) \text {; } \\
& \text { Repeat step } 2 \text { after coding }(j+12)^{\text {th }} \text { frame; } \\
& \text { ENDIF }
\end{aligned}
$$$$
\text { ELSE }
$$$$
\text { ENDIF }
$$

\section{EXPERIMENTAL RESUltS}

We have implemented the proposed algorithm using the H.264/AVC verification model JM11.0 [16] for coding

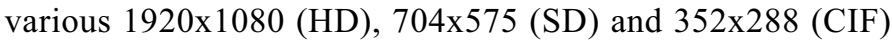
video sequences. TABLE I lists the encoder setup in testing these video sequences.

For testing the dynamic computationally-scalable performance, the first 20 frames of each sequence were coded by H.264 lossless SEA+PDS (i.e. ME state 1) without any computational constraint. Let $C_{U}$ be the average number of ME operations to code a frame by this un-constrained H.264 lossless SEA+PDS. From the $21^{\text {st }}$ frame onwards, ME computational constraint is imposed in which the computational budget, $C_{T}$, is set to be $\alpha C_{U}$, where $0<\alpha<1.0$. 
TABLE I

SETUP IN TESTING SEQUENCES OF DIFFERENT RESOLUTIONS

\begin{tabular}{|c|c|c|c|}
\hline & \multicolumn{3}{|c|}{ Video Resolution } \\
\hline & $1920 \times 1080$ & $704 \times 576$ & $352 \times 288$ \\
\hline $\begin{array}{l}\text { Sequences } \\
\text { tested }\end{array}$ & $\begin{array}{l}\text { Crowd Run, } \\
\text { Park Joy, } \\
\text { Pedestrian, } \\
\text { Rush hour, } \\
\text { Tractor }\end{array}$ & $\begin{array}{l}\text { City, } \\
\text { Crew, } \\
\text { Harbor, } \\
\text { Ice, } \\
\text { Soccer }\end{array}$ & $\begin{array}{c}\text { Akiyo, } \\
\text { Hall, } \\
\text { Mobile, } \\
\text { Mother } \\
\text { Daughter, News }\end{array}$ \\
\hline $\begin{array}{l}\text { No. of ref. } \\
\text { frames }\end{array}$ & 3 & 3 & 5 \\
\hline Search range & 64 & 32 & 16 \\
\hline $\begin{array}{l}\text { Common } \\
\text { settings }\end{array}$ & \multicolumn{3}{|c|}{$\begin{array}{l}\text { Quantization parameter for I and P slice was } 28 \text {. All } \\
\text { seven MB and sub-MB partition sizes were enabled. } \\
\text { Median predictors were used as the initial search centre, } \\
\text { and sub-pixel ME was disabled. Default rate-distortion } \\
\text { optimized mode decision was employed and all video } \\
\text { sequences were encoded in IPPP...format. }\end{array}$} \\
\hline
\end{tabular}

Fig. 4, Fig. 5 and Fig. 6 show the computational performance of the proposed algorithm under different computational constraints $(\alpha=0.3,0.5,0.7)$ and unconstrained H.264 lossless SEA+PDS in coding some HD, $\mathrm{SD}$ and CIF resolution sequences respectively. As shown in these figures, when less computational budget is allocated (i.e. with smaller $\alpha$ ), it takes more time for the proposed algorithm to reach the budget requirement. In particular, for HD sequences tested, the time required to reach budget requirement ranges from 6 to 90 frame time when $\alpha$ changes from 0.7 to 0.3 . Also, it is observed that during constrained $\mathrm{ME}$, the computational complexity can be kept relatively stable for sequences with low or regular motion activities, e.g. Crowd Run (HD) and Mother Daughter (CIF). For sequences with much and irregular motion activities (e.g. Soccer (SD)) or with pan-scan (e.g. City (SD)), the computational complexity fluctuates during constrained ME. It is because for such sequences, the video characteristics, such as the texture, MV field and etc., changes rapidly and so the current search strategy may not meet such characteristics well in reducing the computational complexity. For example, assuming the ME algorithm is now operating at $\mathrm{ME}$ (state, sub-state $)=(5,1)$. If the video suddenly has more violent motion activities, the length of $\mathrm{MV}$ found in the temporal nearest reference frame may become longer. If the ME process still operates at the same ME (state, sub-state), the computational complexity may increase beyond the budget requirement and the closed-loop control will drive the ME process to operate at higher ME (state, sub-state) in order to keep the computational complexity within the allocated budget. Similar situations may occur from time to time during constrained $\mathrm{ME}$ for these kinds of video sequences and hence we observe obvious fluctuations in ME computational complexity.

TABLE II shows the computational budget utilization of the proposed algorithm under different computational constraints $(\alpha=0.3,0.5,0.7)$ in coding HD, SD and CIF resolution sequences. Here, once the computational budget requirement has been reached, our algorithm can effectively keep the average budget utilization to be in the range of $0.88-0.93$ though there might be some instantaneous fluctuation in computational complexity due to varying video characteristics.

TABLE III shows the PSNR performance while TABLE IV shows the bit rate performance of the proposed algorithm under different computational constraints $(\alpha=0.3,0.5,0.7)$ and un-constrained H.264 lossless SEA+PDS in coding HD, $\mathrm{SD}$ and CIF resolution sequences. It is observed that when $\alpha$ increases (or more computational budget is allocated), the compressed video quality improves. In particular, for HD video sequences, the average decrease in PSNR (as compared to that of full search), $\triangle \mathrm{PSNR}$, reduces gradually from $0.068 \mathrm{~dB}$ to $0.019 \mathrm{~dB}$ while the average increase in bit rate (as compared to that of full search), $\Delta$ Bit-rate, reduces gradually from $3.35 \%$ to $1.68 \%$ when $\alpha$ changes from 0.3 to 0.7 .

TABLE II

AVERAGE BUDGET UTILIZATION OF THE PROPOSED ALGORITHM UNDER DIFFERENT COMPUTATIONAL CONSTRAINTS $(\alpha=0.3,0.5,0.7)$ IN CODING HD, SD AND CIF RESOLUTION SEQUENCES

\begin{tabular}{lccc}
\hline \hline & \multicolumn{3}{c}{ Average budget utilization $(\boldsymbol{\gamma})$ when } \\
& $\boldsymbol{\alpha}=\mathbf{0 . 3}$ & $\boldsymbol{\alpha}=\mathbf{0 . 5}$ & $\boldsymbol{\alpha}=\mathbf{0 . 7}$ \\
\hline HD Sequences & 0.93 & 0.91 & 0.92 \\
SD Sequences & 0.92 & 0.91 & 0.92 \\
CIF Sequences & 0.88 & 0.93 & 0.93 \\
\hline
\end{tabular}

\section{Conclusion}

In this paper, we have proposed a computationally-scalable ME algorithm for H.264/AVC video coding. By dynamic adjusting the search strategy to be used via a closed-loop control, the proposed algorithm can control the ME process to be completed within a pre-allocated computational budget while the compressed video quality will be scaled up (down) smoothly when more and more (less and less) computational budget is allocated. We have tested the proposed algorithm to code various $\mathrm{HD}, \mathrm{SD}$ and $\mathrm{CIF}$ video sequences under different computational constraints $(\alpha=0.3,0.5,0.7)$. Experimental results show that the average computational complexity during the constrained ME is $88 \%-93 \%$ of the allocated budget and at the same time, the worst case degradation of PSNR and increase in bit rate as compared to that of full search are $0.068 \mathrm{~dB}$ and $3.67 \%$ respectively.

Compared with those computationally-scalable $\mathrm{ME}$ algorithms as proposed in [10]-[13] in which the ME computational budget can be exactly met, our proposed algorithm cannot guarantee the computational budget is exactly $100 \%$ utilized in coding each frame, but our algorithm has the ability of trying to achieve better compressed video quality by dynamically adjusting the search strategy to be used. Furthermore, compared with those algorithms as proposed in [14] and 15] in which dynamic allocation of computational resources is done at $\mathrm{MB}$ level, our proposed algorithm employs a closed-loop control at frame level for dynamically adjusting the search strategy. 

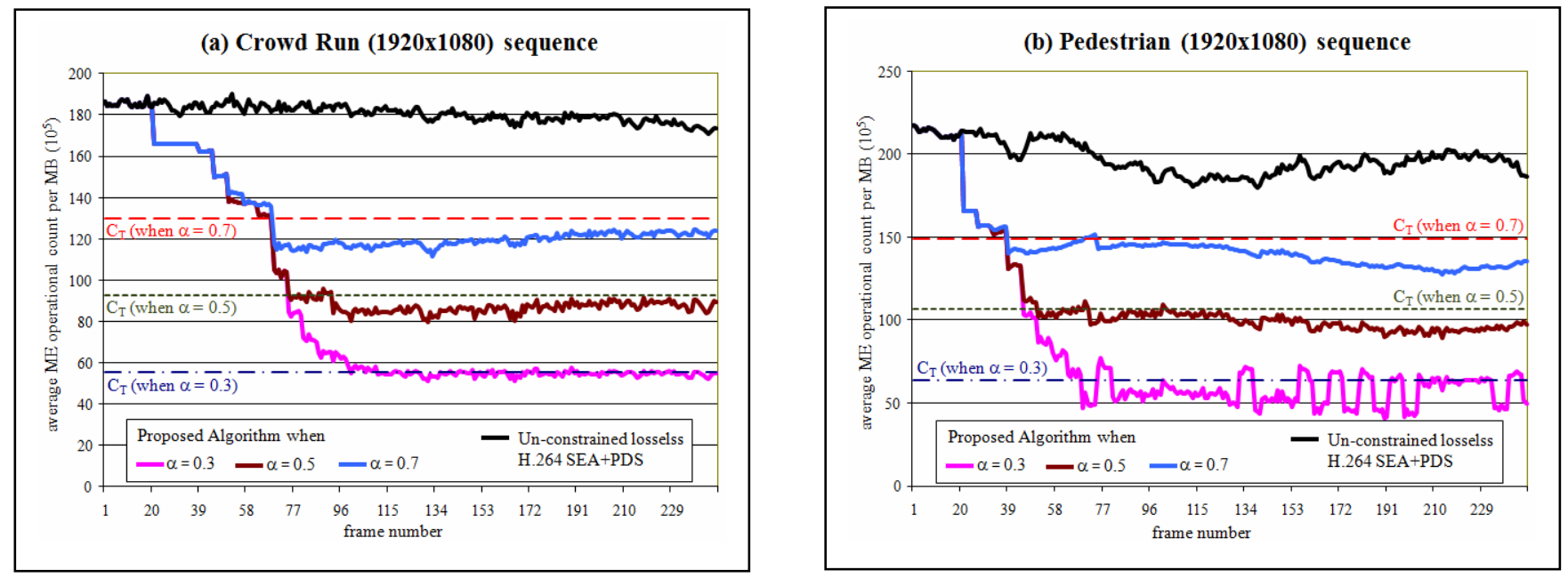

Fig. 4. Computational performance of the proposed algorithm under different computational constraints $(\alpha=0.3,0.5,0.7)$ and un-constrained $H .264$ lossless SEA+PDS in coding 1920x1080 sequences (a) Crowd Run (b) Pedestrian.
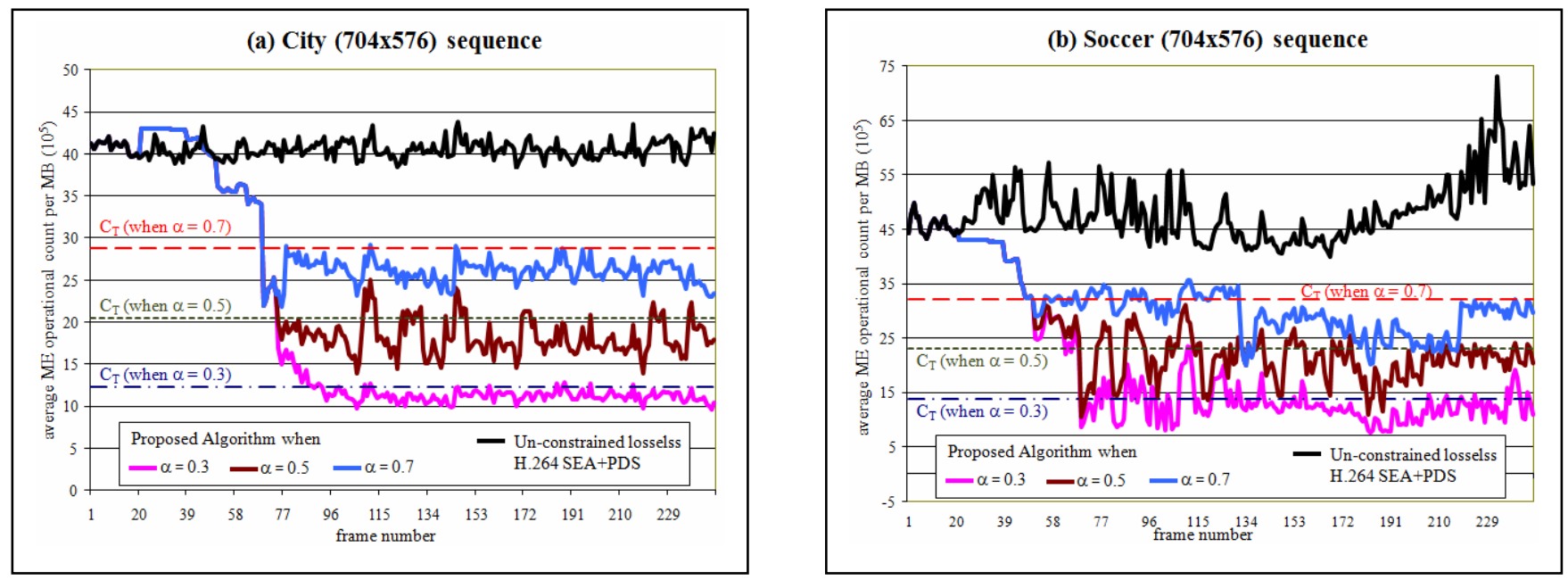

Fig. 5. Computational performance of the proposed algorithm under different computational constraints $(\alpha=0.3,0.5,0.7)$ and un-constrained $\mathrm{H} .264$ lossless SEA+PDS in coding 704x576 sequences (a) City (b) Soccer.
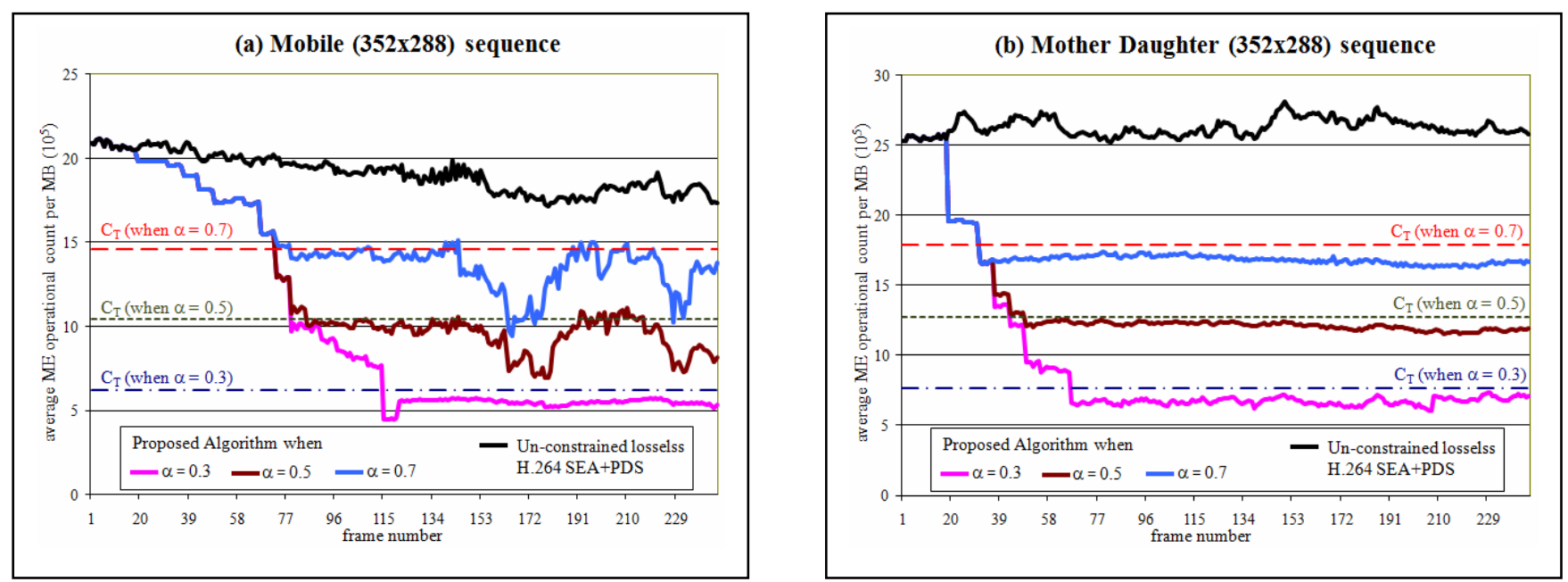

Fig. 6. Computational performance of the proposed algorithm under different computational constraints $(\alpha=0.3,0.5,0.7)$ and un-constrained $\mathrm{H} .264$ lossless SEA+PDS in coding 352x288 sequences (a) Mobile (b) Mother Daughter. 
TABLE III

PSNR PERFORMANCE OF THE PROPOSED ALGORITHM UNDER DIFFERENT COMPUTATIONAL CONSTRAINTS $(\alpha=0.3,0.5,0.7)$ AND UN-CONSTRAINED H.264 LOSSLESS SEA+PDS IN CODING 1920X1080, 704X576 AND 352X288 SEQUENCES (PSNR and $\triangle$ PSNR: $\mathrm{dB}$ )

\begin{tabular}{|c|c|c|c|c|c|c|c|}
\hline \multirow[t]{2}{*}{ Sequence } & \multicolumn{2}{|c|}{$\alpha=0.3$} & \multicolumn{2}{|c|}{$\alpha=0.5$} & \multicolumn{2}{|c|}{$\alpha=0.7$} & \multirow{2}{*}{$\begin{array}{c}\text { H.264 lossless } \\
\text { SEA+PDS } \\
\text { PSNR } \\
\end{array}$} \\
\hline & PSNR & $\Delta$ PSNR & PSNR & DPSNR & PSNR & DPSNR & \\
\hline \multicolumn{8}{|l|}{$1920 \times 1080$} \\
\hline Park Joy & 33.91 & -0.05 & 33.93 & -0.03 & 33.94 & -0.02 & 33.96 \\
\hline Pedestrian & 39.24 & -0.05 & 39.25 & -0.04 & 39.27 & -0.02 & 39.29 \\
\hline Rush hour & 40.85 & -0.05 & 40.87 & -0.03 & 40.90 & 0.000 & 40.90 \\
\hline average & & -0.068 & & -0.046 & & -0.019 & \\
\hline \multicolumn{8}{|l|}{$704 \times 576$} \\
\hline City & 34.80 & -0.12 & 34.82 & -0.10 & 34.85 & -0.07 & 34.92 \\
\hline Crew & 37.14 & -0.05 & 37.18 & -0.01 & 37.19 & 0.00 & 37.19 \\
\hline Harbor & 35.10 & -0.06 & 35.08 & -0.08 & 35.10 & -0.06 & 35.16 \\
\hline Ice & 40.04 & -0.01 & 40.04 & -0.01 & 40.04 & -0.01 & 40.05 \\
\hline Hall & 37.55 & -0.03 & 37.55 & -0.03 & 37.57 & -0.01 & 37.58 \\
\hline Mobile & 33.40 & -0.13 & 33.44 & -0.09 & 33.47 & -0.06 & 33.53 \\
\hline MotherDaughter & 38.29 & -0.05 & 38.33 & -0.01 & 38.35 & 0.01 & 38.34 \\
\hline News & 37.60 & -0.03 & 37.63 & 0.00 & 37.63 & 0.00 & 37.63 \\
\hline average & & -0.048 & & -0.023 & & -0.013 & \\
\hline
\end{tabular}

TABLE IV

BIT-RATE PERFORMANCE OF THE PROPOSED ALGORITHM UNDER DIFFERENT COMPUTATIONAL CONSTRAINTS $(\alpha=0.3,0.5,0.7)$ AND UN-CONSTRAINED H.264 LOSSLESS SEA+PDS IN CODING 1920X1080, 704X576 AND 352X288 SEQUENCES (BIT-RATE: kbps, $\triangle$ Bit-rate: \%)

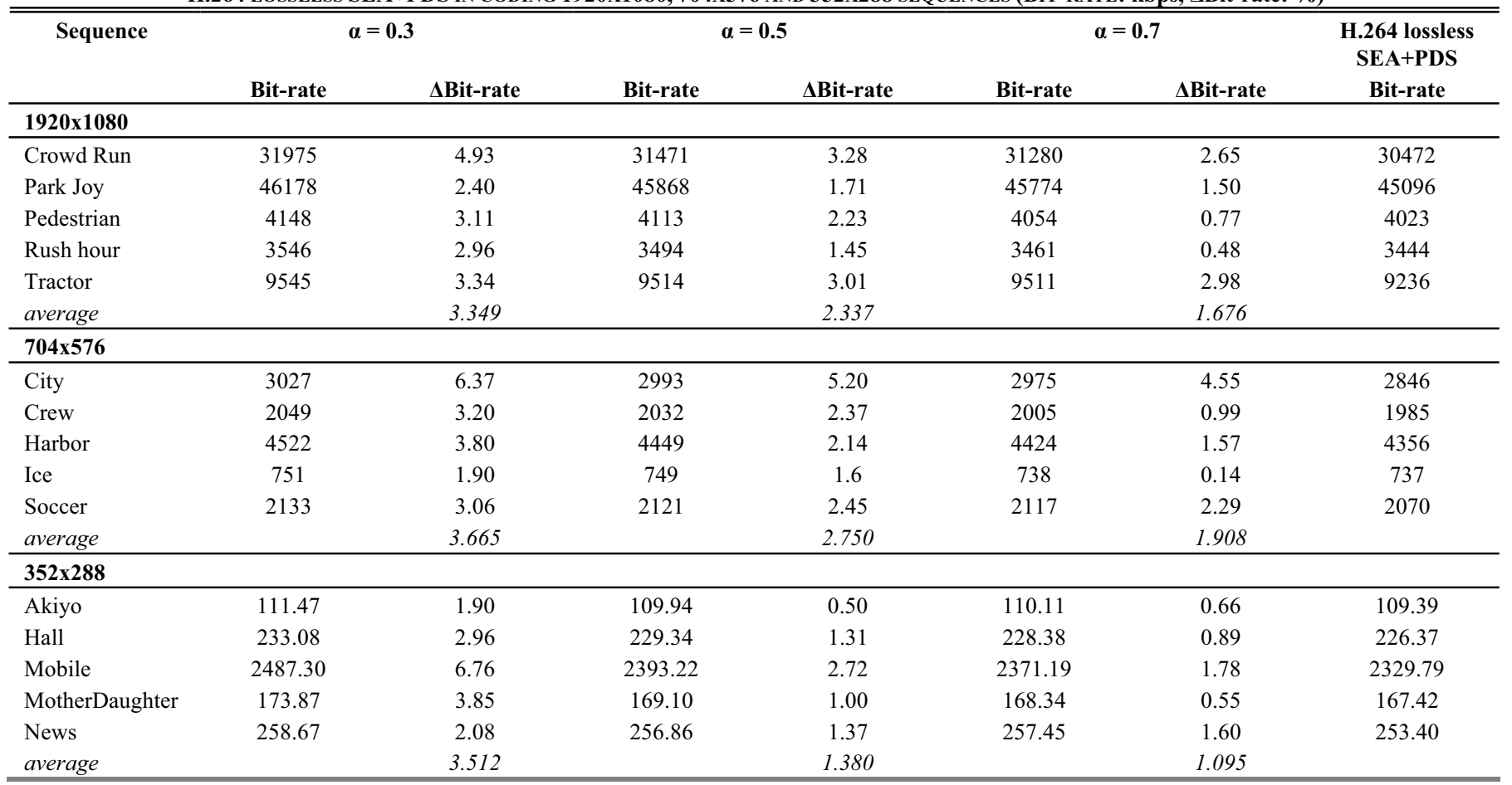

With only slight degradation in compressed video quality during constrained ME, our proposed algorithm can be embraced into the firmware of the multimedia platforms for video products, and allows real-time control of computational budget for various video compression applications.
In our proposed algorithm, there are some pre-defined thresholds (e.g. $T^{R}$ ) used for early termination. In order to achieve even better compressed video quality during the constrained ME, these thresholds should also be adaptively adjusted. As the next step, we will investigate how these 
thresholds can be adaptively adjusted in computationallyscalable ME algorithm, which is a fruitful direction for further research.

\section{REFERENCES}

[1] Information Technology—Coding of audio-visual objects - Part 10: Advanced Video Coding, ISO/IEC Standard 14496-10, 2004.

[2] Thomas Wiegand, Gary J. Sullivan, Gisle Bjontegaard, and Ajay Luthra, "Overview of the H.264/AVC video coding standard," IEEE Trans. on Circuits and Systems for Video Technology, vol. 13, no. 7, pp. 560-576, July 2003.

[3] W. Li and E. Salari, "Successive elimination algorithm for motion estimation," IEEE Trans. Image Processing, vol. 4, no. 1, pp.105-107, Jan. 1995.

[4] ITU-T Recommendation H.263 Software Implementation, Digital Video Coding Group, Telenor R\&D, 1995.

[5] Ko-Cheung Hui, Wan-Chi Siu, and Yui-Lam Chan, "New adaptive partial distortion search using clustered pixel matching error characteristic," IEEE Trans. Image Processing, vol. 14, no. 5, pp. 597-607, May 2005.

[6] Man-Yau Chiu and Wan-Chi Siu, "A new fast full search algorithm for H.264/AVC variable block size multiple reference frames motion estimation," Proceedings of $9^{\text {th }}$ International Conference on Computers, Communications, and Systems, Daegu University, Korea, Nov. 2008

[7] D. Wu, S. Wu, K.P. Lim, F. Pan, Z.G. Li, and X. Lin, "Block INTER mode decision for fast encoding of H.264," Proceedings of IEEE International Conference on Acoustics, Speech, and Signal Processing, vol. 3, pp. 181-184, May 2004.

[8] Liang-Ming $\mathrm{Ji}$ and Wan-Chi Siu, "Reduced computation using adaptive search window size for H.264 multi-frame motion estimation," Proceedings of $14^{\text {th }}$ European Signal Processing Conference, Florence, Italy, 2006.

[9] Krisda Lengwehasatit and Antonio Ortega, "Computationally scalable partial distance based fast search motion estimation," Proceedings of IEEE International Conference on Image Processing, vol. 1, pp. 824827, Sept. 2000.

[10] Zhi Yang, Haixiang Zhang, Jiajun Bu, and Chun Chen, "Complexitycontrollable motion estimation for real-time video encoder," Image Analysis and Recognition - Proceedings of Second International Conference, ICIAR 2005, vol. 3656, pp. 1266-1273, Springer Berlin / Heidelberg, 2005.

[11] Liu Feng, Zhuang Yiqi, and Dai Guoding, "Computational complexity scalable ME algorithm and architecture," Proceedings of IEEE International Conference on Solid-State and Integrated Circuit Technology, pp. 2022-2024, 2006.

[12] Dongming Zhang, Chao Huang, Shouxun Lin, Yanfei Shen, and Lejun $\mathrm{Yu}$, "Complexity scalable motion estimation based on modes preselection in H.264," Proceedings of IEEE International Conference on Signal Processing, vol. 2, pp. 1183-1186, 2004.

[13] Stephan Mietens, Peter H.N. de With, and Christian Hentschel, "Computational-complexity scalable motion estimation for mobile MPEG encoding," IEEE Trans. Consumer Electronics, vol. 50, no. 1, pp. 281-291, 2004.

[14] Zhi Yang, Hua Cai, and Jiang Li, "A framework for fine-granular computational-complexity scalable motion estimation," Proceedings of IEEE International Symposium on Circuits and Systems, vol. 6, pp. 5473-5476, May 2005.

[15] Yih Han Tan, Wei Siong Lee, Jo Yew Tham, and Susanto Rahardja, "Complexity scalable rate-distortion optimization for H.264/AVC," Proceedings of IEEE International Conference on Image Processing, pp. 3397-3400, Nov. 2009.

[16] JVT H.264/AVC Reference Software version JM11.0.

\section{BIOGRAPHIES}

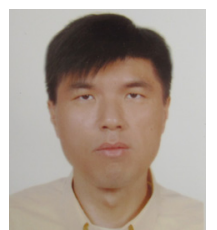

Man-Yau Chiu received B.Eng.(Hons) degree in electrical and electronics engineering from the University of Hong Kong in 1992 and received M.Sc. degree in electronics engineering from the Hong Kong Polytechnic University in 1998. He is now working in Zoran Asia Pacific Ltd. as a senior staff software engineer for MPEG-2 Set-top box (STB) embedded software development. Currently, he is studying in the Hong Kong Polytechnic University for engineering doctorate degree. His research interests include fast motion estimation and scalable video coding.

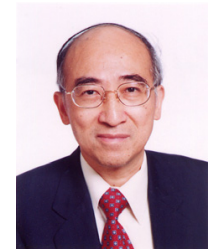

Wan-Chi Siu (S'77-M'77-SM'90) received the Associateship from The Hong Kong Polytechnic University and the MPhil degree from The Chinese University of Hong Kong in 1975 and 1977, respectively, and the Ph.D. Degree from Imperial College of Science, Technology \& Medicine, London, UK, in October 1984. He was with The Chinese University of Hong Kong as a Tutor and later as an Engineer between 1975 and 1980. He then joined The Hong Kong Polytechnic University as a Lecturer in 1980. He was promoted to Senior Lecturer, Principle Lecturer, and Reader in 1985, 1987 and 1990, respectively, and has been Chair Professor of the Department of Electronic and Information Engineering since 1992. He is now Director of the Centre for Multimedia Signal Processing of the same university.

Professor Siu is an expert and a popular speaker in Digital Signal Processing, specializing in fast algorithms and video coding. His research interests also include transforms, image processing, and the computational aspects of pattern recognition and wavelets. He teaches Hi-Tech subjects, such as video technologies and digital signal processing to BEng(Hons) and MSc students, with remarkable support and evaluation from students/graduates. He has successfully supervised over $10 \mathrm{PhD}$ degree and 11 MPhil degree graduates. $\mathrm{He}$ frequently makes open presentations and has published 380 research papers, over 150 of which appeared in international journals, such as IEEE Transactions. His work on fast computational algorithms (such as DCT) and motion estimation algorithms have been well received by academic peers, with good citation records, and a number of which are now being used in hi-tech industrial applications, such as modern video surveillance and video codec design for HDTV systems of some million dollar contract consultancy works.

Professor Siu has been/was Guest Editor, Associate Editor and Member of editorial board of a number of journals, including IEEE Transactions on Circuits and Systems, Pt.II, Pattern Recognition, Journal of VLSI Signal Processing Systems for Signal, Image, Video Technology, the EURASIP Journal on Applied Signal Processing, in addition to other journals. He is an editor of the book, 'Multimedia Information Retrieval and Management', Springer Berlin Heidelberg 2003. He is also the recipient of a number of awards, including the Distinguished Presenter Award (1997, HK), IEEE Third Millennium Medal (2000, USA), the Best Teacher Award (2003, HK), the Outstanding Award in Research (2003, HK), Plaque for Exceptional Leadership (2003, IEEE SPCB, USA), and Honorable Mention Winner Award (Pattern Recognition, 2004, USA). He has been a keynote/invited speaker and key organizer of many international conferences (such as IEEE ICNNSP'08 (keynote speaker, China), IEEE ICICS'07 (invited speaker, Singapore), IEEE CPM'2002 (keynote speaker, Taiwan), IEEE MMSP'08 (General Co-Chair, Australia) and some IEEE society sponsored flagship conferences: ISCAS'97 (technical program co-chair) and ICASSP'2003 (general chair). He is now the General Chair of the IEEE International Conference on Image Processing (ICIP'2010)) to be held in Hong Kong in September 2010. Between 1991 and 1995, Professor Siu was a member of the Physical Sciences and Engineering Panel of the Research Grants Council (RGC), Hong Kong Government, and in 1994, he chaired the first Engineering and Information Technology Panel of the Research Assessment Exercise (RAE) to assess the research quality of 19 Cost Centers (departments) from all universities in Hong Kong. 